Portland State University

PDXScholar

7-2014

\title{
Passive Localization of Noise-Producing Targets Using a Compact Volumetric Array
}

John Gebbie

Portland State University

Martin Siderius

Portland State University, siderius@pdx.edu

Peter L. Nielsen

James Miller

Follow this and additional works at: https://pdxscholar.library.pdx.edu/ece_fac

Part of the Electrical and Computer Engineering Commons Let us know how access to this document benefits you.

\section{Citation Details}

Gebbie, J., Siderius, M., Nielsen, P. L., \& Miller, J. (2014). Passive localization of noise-producing targets using a compact volumetric array. Journal Of The Acoustical Society Of America, 136(1), 80-89. doi:10.1121/1.4881917

This Article is brought to you for free and open access. It has been accepted for inclusion in Electrical and Computer Engineering Faculty Publications and Presentations by an authorized administrator of PDXScholar. Please contact us if we can make this document more accessible: pdxscholar@pdx.edu. 


\title{
Passive localization of noise-producing targets using a compact volumetric array
}

\author{
John Gebbie and Martin Siderius ${ }^{\text {a) }}$ \\ Northwest Electromagnetics and Acoustics Research Laboratory, Department of Electrical and Computer \\ Engineering, Portland State University, 1900 SW 4th Avenue, Suite 160, Portland, Oregon 97201 \\ Peter L. Nielsen and James Miller \\ Centre for Maritime Research and Experimentation, Viale S. Bartolomeo 400, La Spezia, Italy
}

(Received 8 January 2014; revised 18 April 2014; accepted 23 May 2014)

\begin{abstract}
A technique is presented for passively localizing multiple noise-producing targets by crosscorrelating the elevation beams of a compact volumetric array on separate bearings. A target's multipath structure inherently contains information about its range; however, unknown, random noise waveforms make time separation of individual arrivals difficult. Ocean ambient noise has previously been used to measure multipath delays to the seabed by cross-correlating the beams of a vertical line array [Siderius, Song, Gerstoft, Hodgkiss, Hursky, and Harrison, J. Acoust. Soc. Am. 127, 2193-2200 (2010)], but this methodology has not been applied to distant noise sources having non-vertical arrivals. The technique presented in this paper uses a compact volumetric array mounted to an autonomous underwater vehicle to measure the three-dimensional directionality and time delays of multipath arrivals, while adaptively rejecting clutter and multi-target interference. This is validated with experimental results in a shallow ocean environment in which a small workboat maneuvered in the vicinity. Short ranges could be estimated reliably using straight ray paths, but longer ranges required accounting for ray refraction. (C) 2014 Acoustical Society of America. [http://dx.doi.org/10.1121/1.4881917]
\end{abstract}

PACS number(s): 43.30.Cq, 43.30.Zk, 43.30.Re, 43.30.Wi [AMT]

Pages: $80-89$

\section{INTRODUCTION}

This article presents a passive multi-target localization technique that uses a compact volumetric array to decompose the multipath structure arriving on a single bearing, which enables direct measurement of multipath time delays that support target ranging. The hardware and the signal processing requirements needed for this technique lend well to deployment from mobile platforms such as autonomous underwater vehicles (AUVs). Many common ocean waveguides support several ray paths between a source and receiver (known as eigenrays or arrivals), which are collectively referred to as the multipath structure. Since the multipath structure changes as a function of target range, measuring the interference pattern due to multipath can be informative of target range (Thode, 2000; Rakotonarivo and Kuperman, 2012). Multipath interference is the result of time delays between arrivals causing constructive and destructive interference at different frequencies (Harrison, 2011). It has been the subject of much study because the pattern often has low sensitivity to waveguide parameters (Weston and Stevens, 1972). The technique presented in this article bypasses the interference pattern and directly measures the time delays between arrivals by cross correlating beams steered at multipath arrivals. It is based on a recent passive fathometry technique that measured multipath of surface noise. While this approach is presented in the context

\footnotetext{
a) Author to whom correspondence should be addressed. Electronic mail: siderius@pdx.edu
}

of target localization, it also has implications for ocean tomography and geoacoustic inversion.

The rest of this article is organized as follows. Section II gives background on related work. Section III provides a description of the cross-beam correlation methodology. Section IV describes the GLASS' 12 experiment. Section V presents the target localization results from that experiment.

\section{BACKGROUND}

To separate arrivals in time, one approach is to use short-duration waveforms and source receiver geometries that naturally separate rays (Holland and Osler, 2000). Many marine mammals also use short-duration waveforms. For example, whale clicks are impulsive and time delays between distinct multipath arrivals have been shown to provide estimates of animal locations (Nosal and Neilfrazer, 2006; Tiemann et al., 2006; Mathias et al., 2013). The problem is less straightforward when the waveform is broadband noise, but a class of techniques built on the concept of "pulse compression" have been shown to be an effective method of estimating time-delays. Pulse compression is typically based on the generalized cross correlation algorithm (Carter and Knapp, 1976). It can also be used to estimate multipath time delays and has been studied extensively from a theoretical perspective for target range estimation (Hahn, 1975; Hamilton and Schultheiss, 1992; Badriasl et al., 2011).

Arrival angles also are functionally dependent on the environment, and they are used in acoustic tomography experiments, such as Aulanier et al. (2013). This was based on a double-beamforming measurement concept introduced 
by Roux et al. (2008) involving a vertical receiver array and a vertical source array. The ambient noise field has also been shown to provide tomographic information, as recently demonstrated by Leroy et al. (2012) and Lani et al. (2013), extracting coherent wavefronts using multiple vertical line arrays.

Multipath coherence gives rise to an interference pattern that depends on range. Traditionally this has been treated as a nuisance factor, but recently, it has been favorably utilized by a modified conventional beamformer to increase array gain (Rouseff and Zurk, 2011). However, multipath coherence can negatively affect adaptive beamforming algorithms that are based on eigendecomposition. A variety of methods have been developed for breaking the coherence [see Van Trees (2002a) for a summary]. An approach taken by Koch and Knobles (2005) for geoacoustic inversion using noise from a nearby ship of opportunity was to look for correlations between sub-apertures of a horizontal line array, thus avoiding the coherence problem. Later work by Stotts et al. (2010) used the same technique but to simultaneously invert for the ship location and geoacoustic properties.

While multipath coherence can cause problems for adaptive beamforming, this feature was exploited for geoacoustic inversion in the passive fathometer formulation that used surface-generated noise (such as from wind and breaking waves) as a source of opportunity. An algorithm based on conventional beamforming was introduced by Siderius et al. (2006) and later refined by Gerstoft et al. (2008), Harrison and Siderius (2008), and Means and Siderius (2009). The two-way travel time from the array to the seabed was measured by beamforming at the end fire directions of a vertical line array to isolate the downward traveling noise and the seabed reflections. Interestingly, switching to adaptive beam forming (Siderius et al., 2010) improved time delay estimates by reducing interference from nonvertically traveling waves. In the next section, a similar approach is taken to measure time delays between non-vertically traveling multipath arrivals originating from a distant surface noise source.

\section{METHODS}

In the case of the passive fathometer, the vertical array was oriented in such a way that the ends of the array naturally pointed at the source (surface noise) and the multipath reflection (the seabed), while all other interference arrived mainly from broadside (i.e., horizontally). The vertical line array geometry is thus well suited to this type of distributed source, but applying it to a distant source requires forming beams at other elevation angles to match the multipath arrivals. While this is possible with a vertical line array, it also results in a conical beam pattern giving it ambiguity in bearing. This means that ambient noise and multipath from multiple targets will overlap in the beamformer output, making analysis of individual targets difficult. A solution is to use volumetric arrays that have apertures in all three spatial dimensions since they can form beams in any direction in bearing and elevation.

Cross-beam correlation operates on two arrivals, so this discussion begins with a simplified model of a waveguide that includes only the first two arrivals. The spectral value at range $r$ and frequency $\omega$ can be approximated with the first arrival normalized to one as

$$
S \approx e^{-i \omega t_{1}}+R e^{-i \omega t_{2}},
$$

in which $t_{n}$ is the time offset of the $n$th arrival, $R=|R| e^{i \phi R}$ is the complex amplitude of the second arrival relative to the first that accounts for propagation differences (i.e., additional reflections, losses due to spreading and attenuation, etc.). Note the variables $R$ and $t_{n}$ depend on $r$, and we make the approximation that $R$ is independent of frequency, such as would be the case for a half space seabed. Let the multipath time delay be defined as $\tau_{2,1}=t_{2}-t_{1}$.

The rest of this section discusses how to directly obtain $\tau_{2,1}$ from a noise-producing target by beamforming to isolate the individual terms of Eq. (1), which are then cross-correlated to expose $\tau_{2,1}$ in the time domain. The term $\phi_{R}$ is also measurable with cross-beam correlation, but it is ignored mainly because it is a property of the environment and does not contain much information about the target range.

\section{A. Adaptive beamforming}

The first step is to determine the beams on which the multipaths are arriving. This is accomplished with adaptive beamforming that filters the acoustic data in bearing, elevation, and frequency. It results in a map showing where acoustic energy is being received at the array, and its spectral content. Thus, adaptive beamforming provides a means to determine the direction of a target in bearing and its associated multipath arrivals in elevation. Adaptivity plays an important role in that it can focus in one direction while simultaneously rejecting noise arriving in other directions. As its name implies, adaptive beamforming adapts the beam pattern to the data being processed to improve overall gain in the steering direction. Conceptually, it does this by steering side lobes and nulls in directions that are most advantageous. For example, a loud interferer arriving on a distinct bearing will have a null steered at it, whereas a relatively quiet bearing will get assigned a large side lobe.

The minimum-power distortionless response (MPDR) adaptive beamformer is defined in Van Trees (2002c) as

$$
\mathbf{w}_{\mathrm{MPDR}}=\frac{\mathbf{R}^{-1} \mathbf{v}}{\mathbf{v}^{H} \mathbf{R}^{-1} \mathbf{v}} .
$$

The term $\mathbf{R}$ is the sample-averaged cross-spectral density matrix (CSDM) defined as

$$
\mathbf{R}=\frac{1}{M} \sum_{m=1}^{M} \mathbf{p}_{m} \mathbf{p}_{m}^{H},
$$

in which $\mathbf{p}_{m}$ is a vector of spectral values across all channels for the $m$ th snapshot. A snapshot consists of contiguous time-series data across all channels that is used to estimate spectral values. The total averaging time and snapshot length are important and will be discussed in Sec. IIIC. To 
eliminate strong tonal components (such as engine harmonics from a boat) pre-whitening is used to flatten the spectrum by $\mathbf{p}_{m}=\hat{\mathbf{p}}_{m} /\left|\hat{\mathbf{p}}_{m}\right|$, where $\hat{\mathbf{p}}_{m}$ is the measured spectral value. The plane wave array manifold is

$$
\mathbf{v}(\mathbf{u})=e^{-i k \mathbf{a}^{T} \mathbf{u}}
$$

for which a is the array geometry that specifies the position of each element in a column vector, $\mathbf{u}$ is a unit vector indicating the direction of wave propagation in a column vector, and $k$ is the wave number in units of radians per meter. In the literature, MPDR is sometimes referred to as MVDR (where the V stands for variance); however, strictly speaking the MVDR algorithm (Capon, 1969) requires a priori knowledge of the noise $(\mathrm{N})$ plus interference (I) covariance matrix $\left(\mathbf{K}_{\mathrm{N}+\mathrm{I}}\right)$, whereas MPDR operates directly on the sample-averaged CSDM, R. Equation (2) is a least-squares solution to

$$
\underset{\mathbf{w}}{\operatorname{argmin}} \mathbf{w}^{H} \mathbf{R} \mathbf{w}
$$

which minimizes the power output of the beamformer while satisfying the distortionless constraint

$$
\mathbf{w}^{H} \mathbf{v}=1 \text {. }
$$

The formulation in Eq. (2) requires $\mathbf{R}$ to be full rank. It is often the case in practice that $\mathbf{R}$ has one or more very small eigenvalues, which cause the matrix inversion to become unstable. This can happen for an $N$ element array with fewer than $N$ snapshots, and it is referred to as "snapshot deficiency" (Song et al., 2003). While techniques exist to compensate for this (Menon and Gerstoft, 2013), a common ad hoc technique for stabilizing the matrix inversion is to add a small amount of diagonal loading to $\mathbf{R}$; in this study, a small amount $(-30 \mathrm{~dB}$ relative to the mean spectral power across channels) is used (Van Trees, 2002b). Beyond stabilizing a rank deficient $\mathbf{R}$, diagonal loading can also increase the beamformer tolerance to mismatch errors, such as element location errors or wavefront curvature errors.

\section{B. Cross-beam correlation}

Once multipath arrivals are spatially separated on different elevation beams, the next step is to determine the time delay of their respective signals. The noise signal from the source undergoes different delays based on unique ray trajectories through the waveguide. Cross-correlation can be used to "pulse compress" these coherent broadband noise signals into a broadband pulse in the time domain, which has an offset corresponding to the relative time delay. This shares some conceptual similarity to a matched filter commonly used in active sonar, but instead of a known transmitted waveform, it uses a measured waveform.

In the time domain, cross correlation is computed by sliding a "reference" signal along the time axis while holding the "correlated" signal fixed, taking the product at each offset, and finally integrating. In the frequency domain, this corresponds to conjugating the spectrum of the reference signal and multiplying it by the spectrum of the correlated signal. The cross spectrum of two arbitrary beams can thus be formulated as

$$
\begin{aligned}
C_{\mathrm{c}, \mathrm{r}} & =\left(\mathbf{w}_{c}^{H} \mathbf{p}\right)\left(\mathbf{w}_{r}^{H} \mathbf{p}\right)^{*} \\
& =\mathbf{w}_{c}^{H}\left(\mathbf{p p}^{H}\right) \mathbf{w}_{r},
\end{aligned}
$$

in which $\mathbf{w}_{c}$ is the correlated beam and $\mathbf{w}_{r}$ is the reference beam. The quantity in parenthesis in Eq. (8) is the outer product of a single snapshot; if multiple snapshots are averaged, this is replaced with a sample-averaged CSDM, R. Cross-beam correlation for arbitrary beams is thus defined as

$$
C_{\mathrm{c}, \mathrm{r}}=\mathbf{w}_{c}^{H} \mathbf{R} \mathbf{w}_{r}
$$

Inverse Fourier transforming (with the operator denoted as $\mathcal{F}^{-1}$ ) yields

$$
\Gamma_{\mathrm{c}, \mathrm{r}}(\tau)=\mathcal{F}^{-1}\left[\hat{C}_{\mathrm{c}, \mathrm{r}}(\omega)\right]
$$

The envelope can then be used to eliminate constant phase offsets as

$$
\Gamma_{\mathrm{c}, \mathrm{r}}^{\mathrm{env}}(\tau)=\left|\Gamma_{\mathrm{c}, \mathrm{r}}(\tau)+i \mathcal{H}\left[\Gamma_{\mathrm{c}, \mathrm{r}}(\tau)\right]\right|,
$$

in which $\mathcal{H}$ is the Hilbert transform. Time delays are then obtained by finding the offset of the largest peak as

$$
\tau_{\mathrm{c}, \mathrm{r}}=\underset{\tau}{\operatorname{argmax}}\left[\Gamma_{\mathrm{c}, \mathrm{r}}^{\mathrm{env}}(\tau)\right] \text { for } \tau_{\min }<\tau .
$$

The term $\tau_{\min }$ is an artifact due to white noise leakage and depends on the array geometry, the manifold origin, and the beams being correlated. It is defined as

$$
\tau_{\min }=\max _{n}\left[\mathbf{a}_{n}^{T}\left(\mathbf{u}_{r}-\mathbf{u}_{c}\right) / c\right]+\frac{1}{B_{s}}
$$

in which $B_{s}$ is the effective target bandwidth, and $c$ is the sound speed at the array.

Equation (9) is a generalization of the passive fathometer to beams steered in arbitrary directions for an array of arbitrary geometry. For comparison, the vertical beams of the passive fathometer are $\mathbf{w}_{\text {down }}=\mathbf{w}_{r}$ (the signal traveling down from the surface) and $\mathbf{w}_{\text {up }}=\mathbf{w}_{c}$ (the signal traveling up from the seabed). It is useful to denote the beam having the delayed signal as the correlated beam, which renders time-delays as positive quantities. However, this is merely a matter of convention.

\section{Target localization procedure}

This procedure uses array acoustic data and knowledge of the receiver position in the waveguide to estimate target range. In this scenario, the target is assumed to be at the surface. If additional information about the sound speed profile (SSP) of the waveguide is available, this can be used to improve range estimates. The total acoustic averaging time should be enough to produce a full rank $\mathbf{R}$ but short enough 
so the effects of target motion are minimal; this may be on the order of hundreds of milliseconds depending on the array size.

The first step in the procedure is to use adaptive beamforming to find all arrivals from all targets. Coherence between multipath arrivals can cause problems with adaptive beamforming since coherent signals arriving on distinct beams are represented by a single eigenvector. A way to mitigate this is to reduce the snapshot length in order to break the coherence. As the snapshot length approaches the time delay between multipath arrivals, each arrival will be increasingly mapped to distinct eigenvectors. This has the effect of stabilizing the adaptive beamformer for coherent arrivals originating from a single target. The output of the beamformer is a full three-dimensional map of all arrivals on all bearings and all elevations. For a single target on a single bearing, the multipath arrivals will be distributed on the elevation axis.

The second step is to cross-correlate beams steered at different elevation angles on a single bearing to determine the time delay between arrivals. Unlike the previous step that attempts to eliminate multipath coherence, this step re-averages $\mathbf{R}$ using snapshots that are longer than the multipath time delay, but without changing the total averaging time. The length of the snapshot should be on the order of several times the delay to be measured.

With a surface source in shallow water, the first arrival will usually be traveling downward and will have the highest amplitude, so a method to find the delay between just the first two arrivals is to steer the reference beam at the strongest arrival traveling downward and sweep the correlated beam over all elevation angles on the same bearing. If the correlated beam corresponds to an arrival that is delayed with respect to the arrival in the reference beam, a peak will appear at a positive time offset in the time series corresponding to the delay.

The third step is to use the receiver position in the waveguide, and possibly environmental data as well, to estimate the target range. For a surface target, image theory can be used to compute this time delay in a closed form (Jensen et al., 2011). This assumes a constant water sound speed over depth. However, if data pertaining to the SSP or bathymetry exists, a ray model can be employed to compute the first two eigenray travel times. This same calculation is performed at all ranges for a constant receiver depth and compared to the measured time delay. Ranges at which there is agreement imply a greater likelihood the target is at that range. This is demonstrated with experimental results in Sec. $\mathrm{V}$. Note that ray travel times can have varying degrees of sensitivity to the water SSP. Recent work has been done on travel time sensitivity kernels for range and depth dependent variations (Sarkar et al., 2011), but in this study, we assume range independence.

\section{EXPERIMENT}

This section describes the experimental design used to validate the proposed technique. The GLASS' 12 experiment was conducted by the Center for Maritime Research and

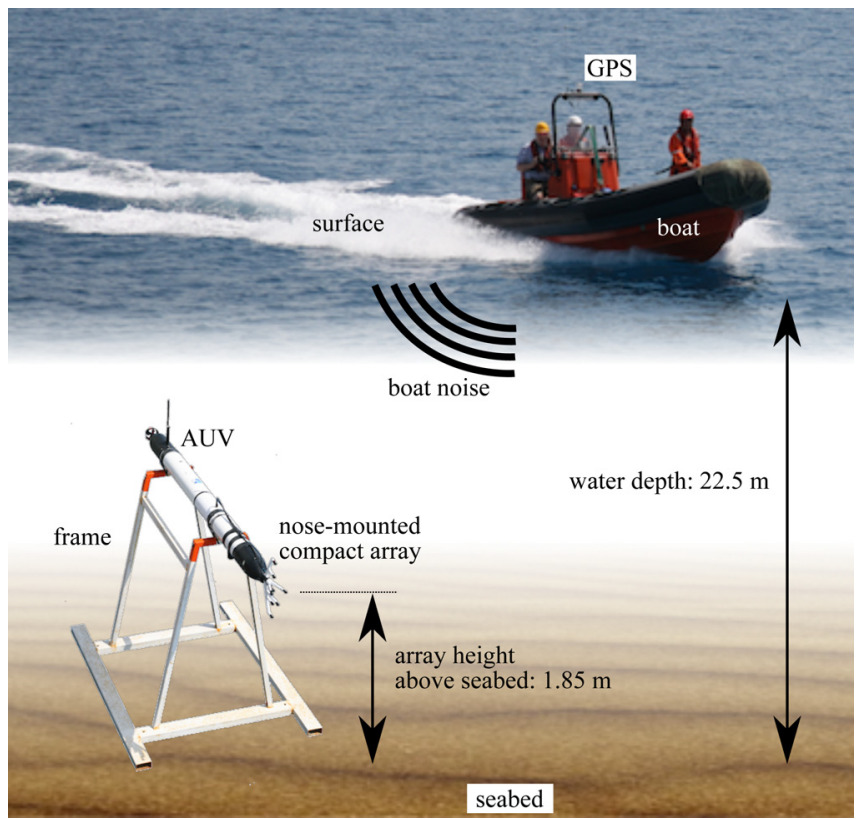

FIG. 1. (Color online) Experimental setup.

Experimentation (CMRE) from the NRV Alliance in July 2012 off the coast of northern Italy near La Spezia. The purpose was to investigate potential uses of an autonomous underwater vehicle (AUV) affixed with a nose-mounted array. The CMRE AUV eFOLAGA was chosen as the "autonomous" platform. Because of technical problems with the ballasting subsystem, acoustic data recordings were collected by mounting the AUV on a rigid frame, as illustrated in Fig. 1. The mooring placed the array approximately $1.85 \mathrm{~m}$ above the seabed, and the overall water depth was approximately $22.5 \mathrm{~m}$, as measured by a towed EdgeTech SB216S sub-bottom profiler. An overview map of the deployment site and regional bathymetry is shown in Fig. 2. Results presented in the paper were recorded at point $\mathrm{G}$. The SSP was measured with a conductivity-temperature-depth (CTD) instrument, and it is shown in Fig. 3. Portions at the

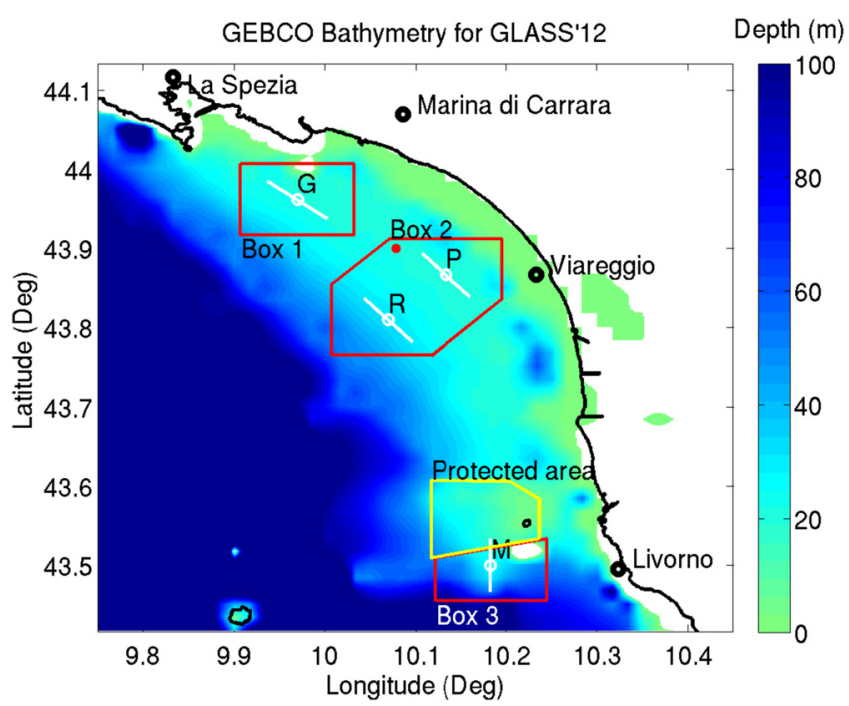

FIG. 2. (Color online) Bathymetry and overview map of experiment site. Data presented in this article were taken at site $\mathrm{G}$. 


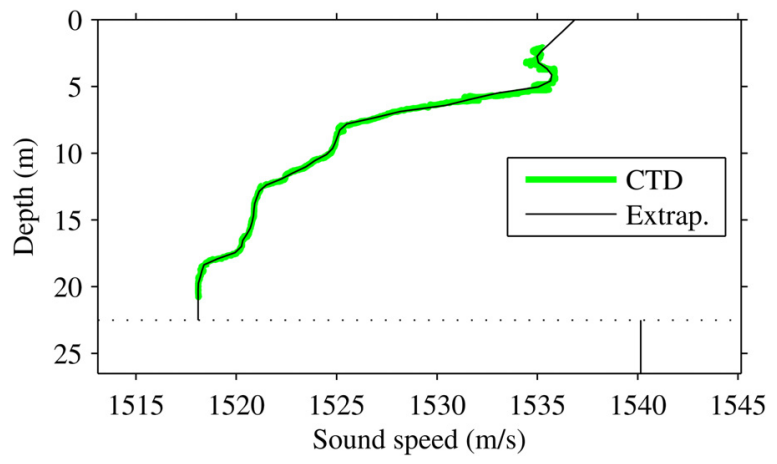

FIG. 3. (Color online) Water sound speed profile (SSP) showing measured and extrapolated parts.

boundaries are extrapolated over depths for which no data was captured due to limitations of the instrument. The extrapolation is explained in Sec. V C. Analysis of core samples from the seabed indicated a mean seabed density of $1.807 \mathrm{~g} / \mathrm{cm}^{3}$ and mean sound speed of $1540.1 \mathrm{~m} / \mathrm{s}$. Attenuation measurements for the seabed were not available, but a nominal value of $0.2 \mathrm{~dB} / \lambda$ was assumed.

The array shown in Fig. 4 was designed and built by CMRE. It consisted of eight elements: five in a vertical configuration and three offset from the center element to form a tetrahedron with the center element. The elements are mounted on a rigid frame providing low element position error. The spacing between the vertical elements, as well as edge lengths of the tetrahedron, was $10 \mathrm{~cm}$. The sample rate of the array was $100 \mathrm{kHz}$. The GPS coordinates of the array position were taken during the deployment.

A small boat (the CMRE workboat) with an outboard engine outfitted with a portable GPS data recorder maneuvered in the vicinity of the array. The speed of the boat was roughly $3 \mathrm{~m} / \mathrm{s}$. Selected portions of the track of the boat are shown in Fig. 5(a). Besides the NRV Alliance, there were few vessels detected in the local region. To simulate a

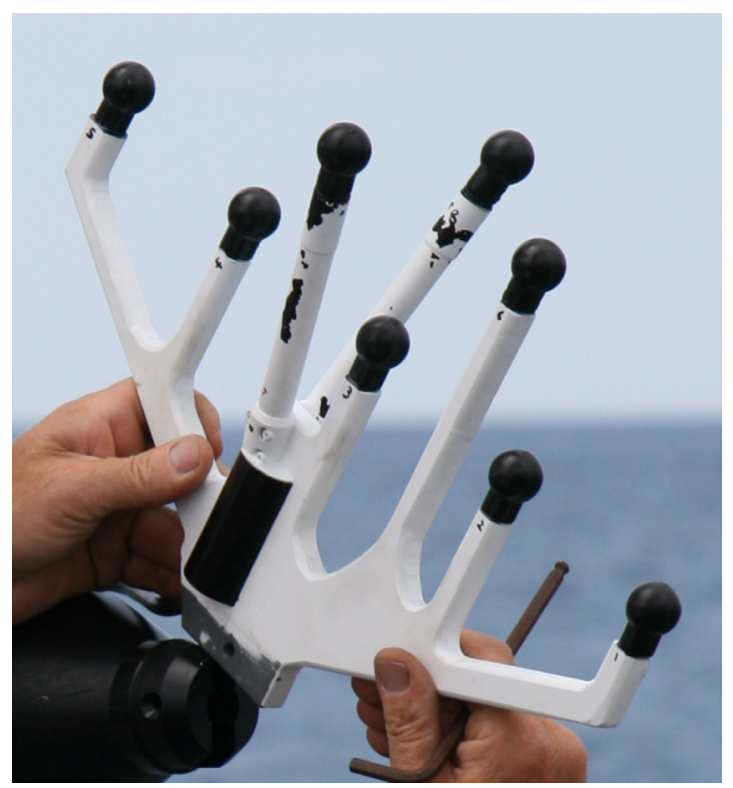

FIG. 4. (Color online) The passive acoustic array consisting of tetrahedral and line sub arrays. This was mounted to the nose of the eFolaga AUV.
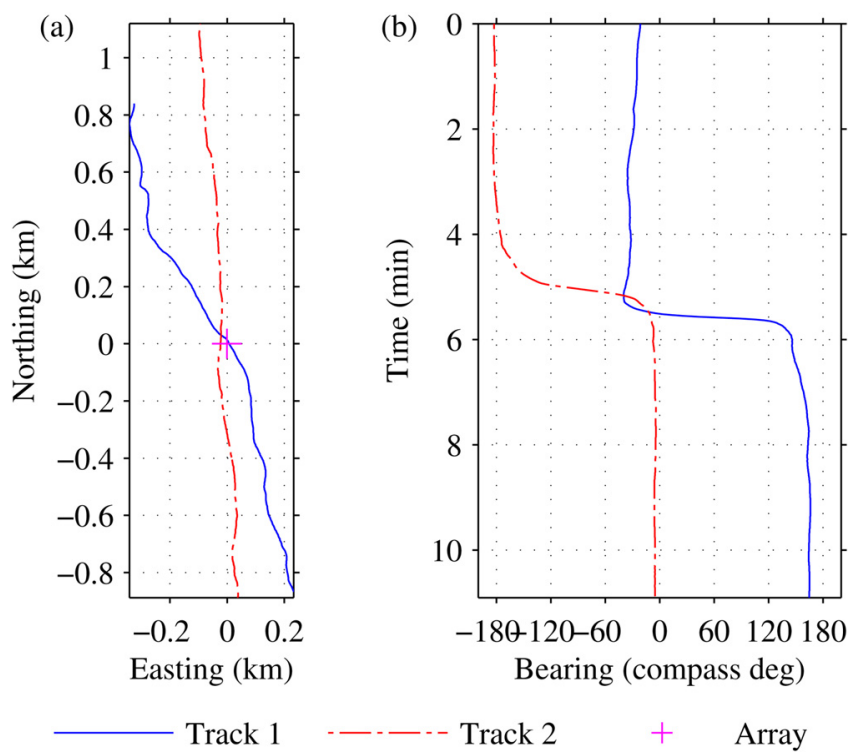

FIG. 5. (Color online) (a) Tracks of the same boat for two passes by the array, taken from GPS records. (b) Compass bearings of the two same two tracks. A multi-target scenario is simulated by adding the acoustic data from each track. This plot shows how they are aligned in time.

multiple target scenario, acoustic data from two boat passes by the array were added together. The time alignment of these two passes, showing the relative bearings of each pass are shown in Fig. 5(b).

\section{A. Predicted multipath structure}

This section describes how the multipath structure was determined from the measured environmental parameters. Bellhop (Porter and Bucker, 1987) is a popular ray tracing tool for analyzing acoustic propagation between two points in an ocean waveguide. Formulating this problem in a ray context has the advantage of being able to clearly see the contributions of each eigenray in terms of both space and time. Bellhop produces an estimate of the full path traversed by the eigenray. It also produces an estimate of the travel time for each eigenray, which depends on the SSP. As a matter of terminology for this article, eigenrays are labeled according to their sequence of boundary interactions; the direct eigenray is " $\mathrm{D}$," the bottom-reflected path is " $\mathrm{B}$," the path reflected first from the bottom then the surface is "BS," etc.

Figure 6 shows a set of eigenrays computed with Bellhop. The paths of these rays can be understood by noting the negative gradient of SSP shown in Fig. 3 (i.e., the trend shows decreasing sound speed with increasing depth). With the source placed at the surface, the first surface bounce is omitted following the same procedure in Holland and Osler (2000). The negative gradient causes rays to bend toward the seabed; an effect that is amplified as rays are launched closer to the horizontal. Note that Fig. 6(c) is the last panel that shows a D and B eigenray. This corresponds to the maximum range for which the $\mathrm{D}$ eigenray exists. The launch angle for the D eigenray at that range is zero (exactly horizontal), meaning that just beyond that range the ray must first reflect off the seabed to be received by the array, which 


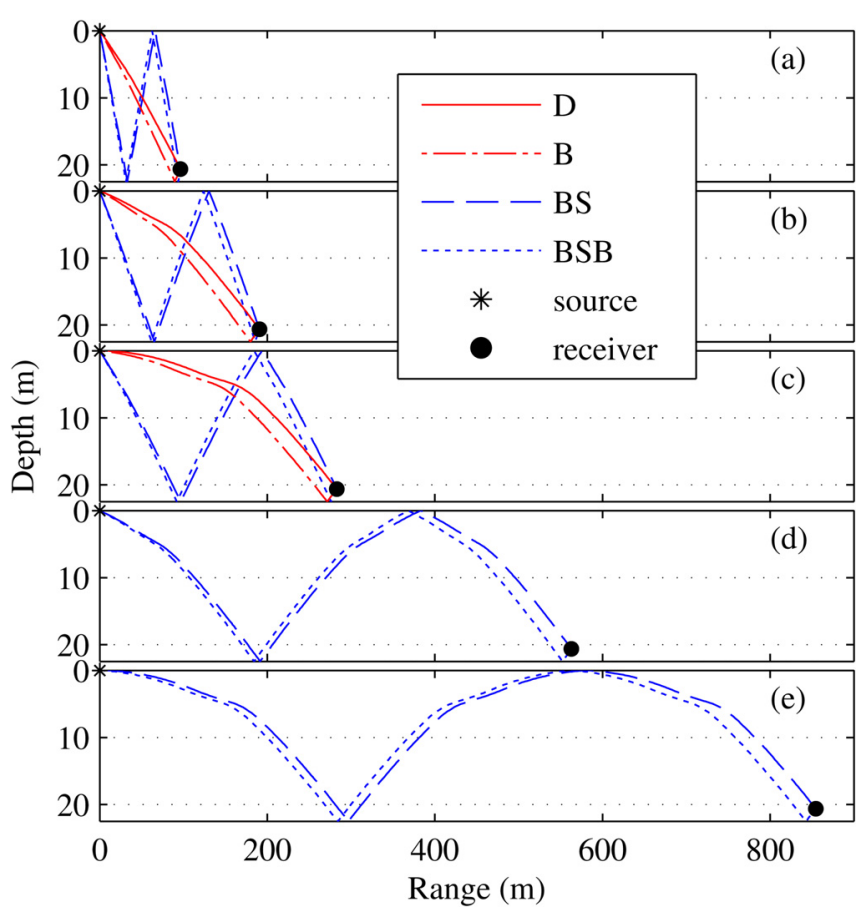

FIG. 6. (Color online) Ray trace using Bellhop. (a)-(e) Only the D, B, BS, and $\mathrm{BSB}$ eigenrays are shown for different source-receiver ranges. Note the culling of the D and B eigenrays as the range approaches $300 \mathrm{~m}$. Panel (c) represents the maximum range of the $\mathrm{D}$ eigenray, which corresponds to a $0^{\circ}$ ray launch angle. Likewise, panel (e) shows the maximum range of the BS eigenray.

essentially culls the D eigenray. The same phenomenon happens for the BS ray; its limiting range is shown in Fig. 6(e).

\section{RESULTS AND ANALYSIS}

This section demonstrates the proposed technique using data from the GLASS'12 experiment and compares this against results derived from measured environmental and GPS data. Section VA demonstrates how adaptive

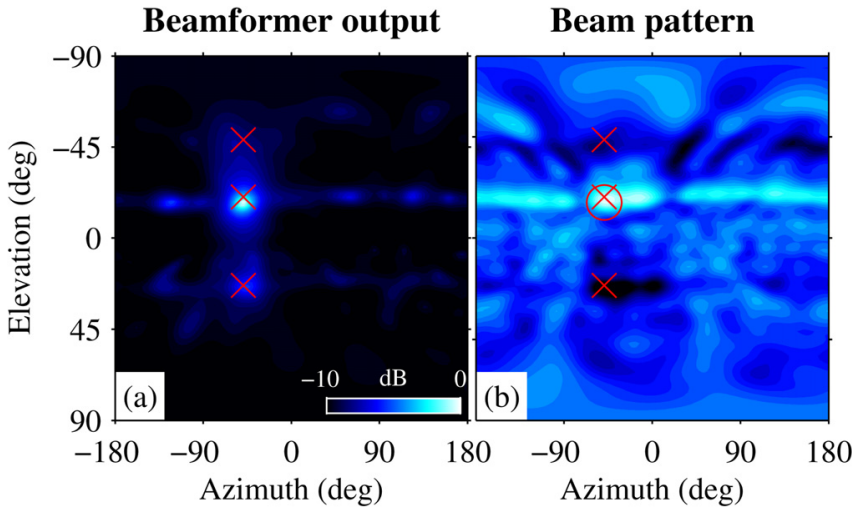

FIG. 7. (Color online) (a) Adaptive beamformer output averaged over $1-35 \mathrm{kHz}$. (b) Beam pattern steered at the strongest arrival traveling downward, averaged over the same band. The $\times$ markings denote expected arrivals from ray tracing, and the [circo] marking is the steering direction.

beamforming can be used to measure both the target bearing and elevation angles of individual multipath arrivals. Section $\mathrm{V} B$ uses the beam directions with cross-beam correlation to measure time delays between the two dominant arrivals. Section V C aggregates time delays over the entire boat run for single- and multi-target scenarios and also illustrates range-dependent multipath features. Section V D interpolates the range from measured time delays.

\section{A. Beamforming to find multipath arrivals}

Volumetric arrays provide the capability of steering beams in any direction, which is used here to determine target bearings as well as the multipath arrival structure that is spread over the elevation angles. In this analysis, CSDMs are computed using $150 \mathrm{~ms}$ of total averaging time, and so the target motion is assumed to be negligible. While the array in Fig. 4 is geometrically comprised of two subarrays, the full eight-element array is used in this analysis. Adaptive

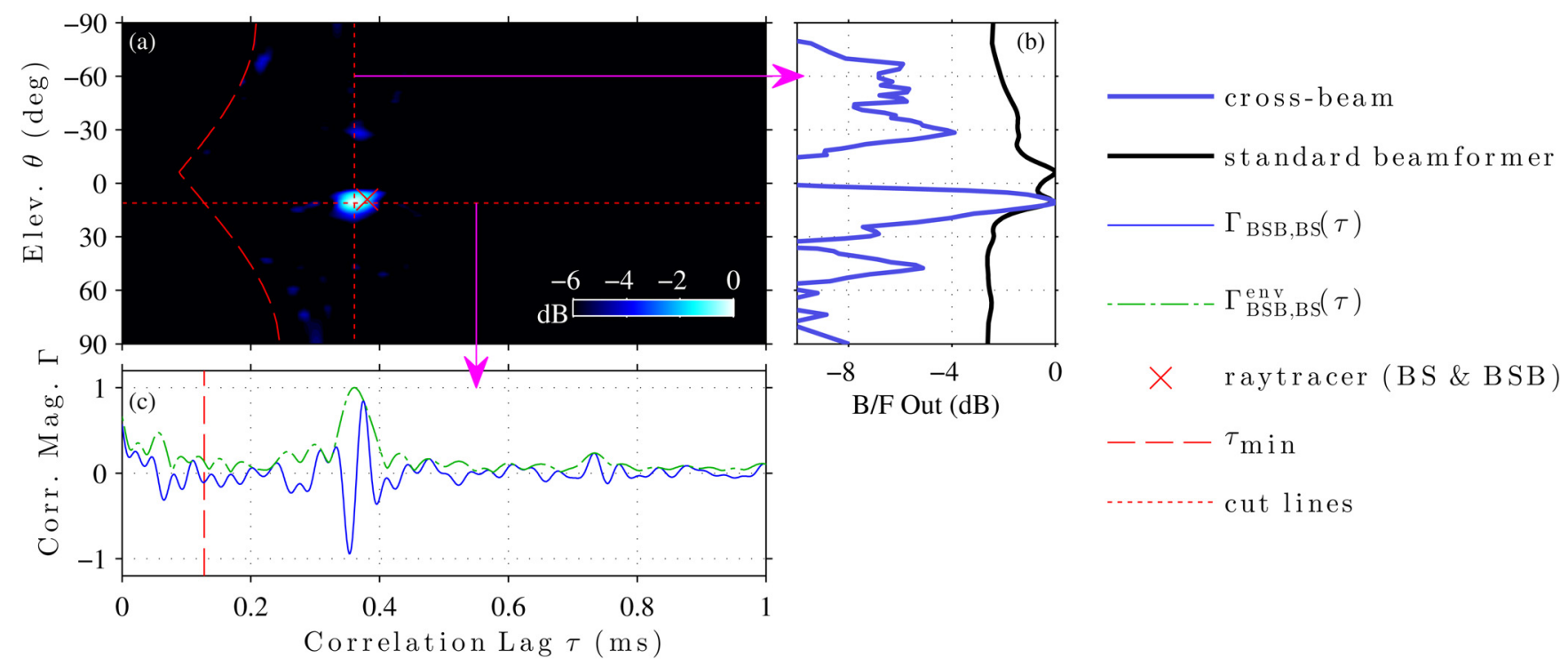

FIG. 8. (Color online) Cross-beam correlation of the strongest arrival traveling downward with other elevation angles along the same azimuthal direction. (a) Correlation power envelope at each elevation angle and time delay. (b) A vertical slice of the cross beamformer taken at the measured multipath delay compared with a standard adaptive beamformer output. (c) Cross beamformer time series output at the measured multipath elevation angle showing the actual waveform and its envelope. 
beamformer outputs are shown in Fig. 7(a) when the boat is at $58 \mathrm{~m}$ range and approaching the array. Snapshot lengths are $0.53 \mathrm{~ms}$, and the predicted time delay between D and B eigenrays at this range is $0.904 \mathrm{~ms}$. A Hann time window is applied to each snapshot during averaging, and snapshots overlap by $50 \%$. Beamformer outputs are averaged over the $1-35 \mathrm{kHz}$ frequency band. Note that the axes in Fig. 7(a) are oriented according to the wave propagation vector, for which negative angles correspond to downward traveling waves and vice-versa. The expected arrival directions are predicted with a ray tracer, and these appear near strong beamformer outputs. Figure $7(\mathrm{~b})$ is the adaptive beam pattern of the strongest beam traveling downward showing nulling of other beams, including multipath arrivals.

\section{B. Measuring time delays with cross-beam correlation}

Once the direction of the strongest arrival traveling downward is determined, that beam is then cross correlated with other elevations on the same bearing to find the beam containing the next multipath arrival to measure its delay. This step uses $10 \mathrm{~ms}$ snapshots in order to bring multipath coherence into the CSDM.

This processing follows the steps in Sec. III B such that in Eq. (9) the reference beam is the strongest beam traveling downward and the correlated beam is steered in different elevation angles looking for correlations. If the correlated beam contains a delayed waveform with respect to the reference beam, the correlation delay will be positive in Eqs. (10) and (11). This is demonstrated in Fig. 8(a) in which the boat is at a distance of $797 \mathrm{~m}$ and is approaching the array. Each row is a separate cross-correlation between that elevation angle and the strongest beam traveling downward, measured at $-5.9^{\circ}$. An annotation shows where the ray model predicts the BSB arrival to be; at $9.02^{\circ}$ and $0.38 \mathrm{~ms}$ after the BS arrival. This agrees with the data in which a clear peak is visible on the $11.06^{\circ}$ beam at a time delay offset of $0.36 \mathrm{~ms}$.

Beyond validation against the ray model, the peak in Fig. 8(a) also matches a peak in the standard adaptive beamformer output, further indicating that it is the multipath arrival from the seabed. This can be seen by cutting through the elevation angles at the measured delay, as shown in Fig. 8(b).

Further analyzing the actual time series on the $11.06^{\circ}$ beam, the cross correlation and its envelope are shown in Fig. 8(c). The shape of the pulse in the non-envelope line is not a sinc function, as might be expected from a rectangular band of frequencies, but rather, it shows a significant amount of skewing. This is due to a phase change picked up from an additional seabed reflection, and it is predictable from the ray tracer using measured environmental parameters and assuming a half space seabed. The envelope operation eliminates this constant phase term and yields a maximal value at the time delay between beams.

The minimum bound on time delay measurements, $\tau_{\min }$, varies as a function of the angular subtense between beams being cross correlated, and it is computed according to Eq. (13). The observed peak width in Fig. 8(c) corresponds to an effective signal bandwidth of $B_{s}=11.3 \mathrm{kHz}$.

\section{Time evolution of cross-beam measurements}

This section analyzes how measured time delays between the first two eigenray arrivals change as the boat maneuvers. By stacking the time series [i.e., Fig. 8(c)] at several steps during the boat pass, a cross-beam correlogram is produced, as shown in Fig. 9. Note that this differs from the more typical usage of correlograms that are obtained by cross correlating two receivers. In contrast, this image shows the cross correlation of two beams steered at different elevation angles on a single bearing. The bearing corresponds to the direction of arrival of boat noise, and it changes as the boat maneuvers. To analyze the multipath originating from a single track, prior knowledge of the boat's bearing was used to restrict the bearing search space. Overlaid on the plots of Fig. 9 is the expected time delay of different arrival pairs as estimated from Bellhop using the measured SSP. Note that the ranges at which these lines terminate correspond to the ranges at which
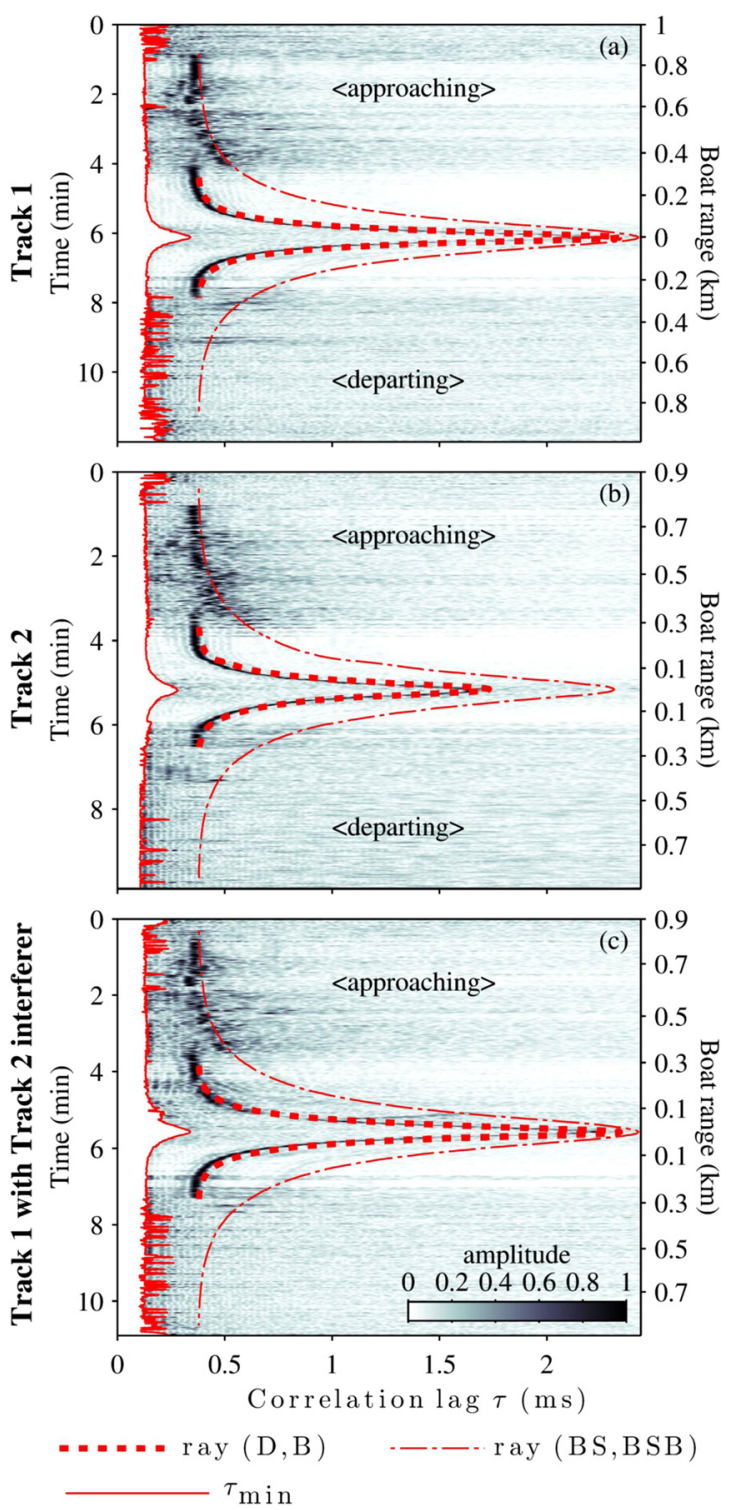

FIG. 9. (Color online) Correlograms of multipath-steered beams with overlays showing model predictions of multipath time delays. (a),(b) Individual boat tracks. (c) Multi-target scenario (sum of acoustic data from both tracks) with cross-beamformer steered at Track 1 . 


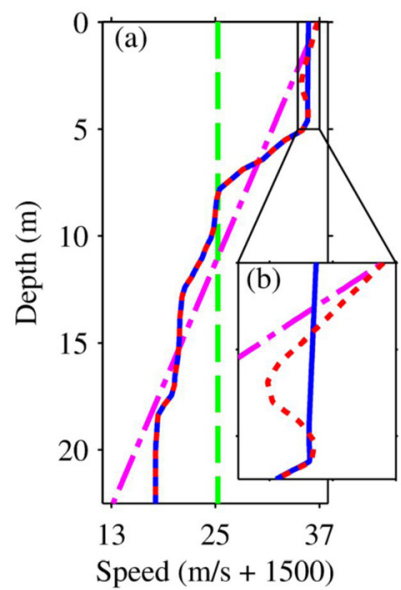

$-\ldots$ iso

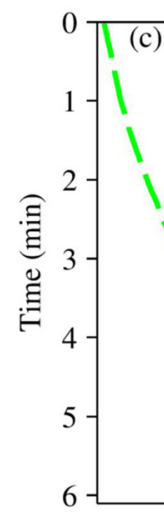

0.1

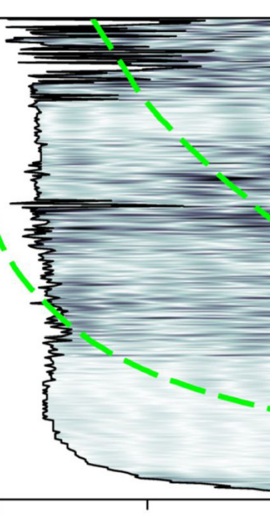

0.2

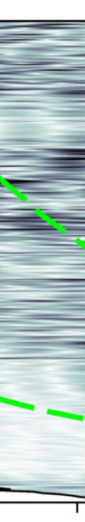

0.3
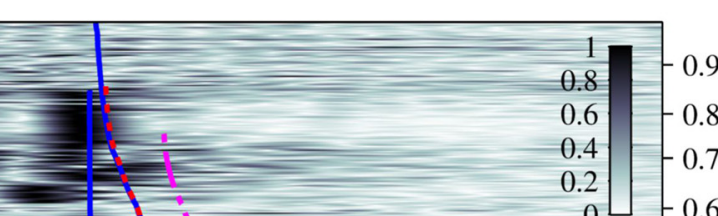

0.6

0.4

$\begin{aligned} & 0.4 \\ & 0.2\end{aligned}-0.7$

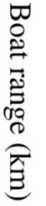

FIG. 10. (Color online) (a),(b) Different SSPs used to configure the ray tracer. (c) Correlogram with overlays showing the multipath delays resulting from each SSP. There are two lines for each SSP that correspond to the delays between each of the two sets of eigenray pairs (D-with-B and BS-with-BSB). Note the ranges at which rays are culled match well with the measured SSP. When the near surface part of the SSP is changed to have a very small gradient, the range at which culling happens changes significantly.

the different eigenray pairs are culled in Fig. 6. This also shows strong agreement with the ranges at which the correlation peaks in the acoustic data change visibility. This is true for both the D-with-B and BS-with-BSB correlations.

Similar results are obtained for Tracks 1 and 2 [comparing Figs. 9(a) and 9(b)]. Track 1 approaches the array from the north and Track 2 from the south. Yet, the BS-with-BSB correlation is visible in both only on the approach, indicating a strong aspect dependence on boat noise radiation. An artificial multi-target scenario is shown in Fig. 9(c) in which acoustic data from Track 1 and Track 2 are added together. The same processing is applied that focuses the cross beamformer on the arrivals from Track 1, and it is seen how adaptive beamforming effectively nulls the Track 2 interferer.

Initially in this study, an isovelocity (constant SSP) model was adopted. This produced accurate predictions of the multipath delay out to about $100 \mathrm{~m}$, but beyond that, the range delay predictions were smaller than observed. These observations became explainable once a ray model was adopted and configured with the measured SSP. The downward refracting profile causes an increase in the delay, and its effect starts to become more pronounced at about the $100 \mathrm{~m}$ range. This is evident by comparing the multipath delays computed using different SSPs to the acoustic data in Fig. 10. The isovelocity model clearly diverges from the acoustic data around this range whereas the other SSPs, which are downward-refracting, produce measurable delays out to farther ranges. The downward refraction causes rays launched near the surface to have steeper angles as they pass through the array depth. This implies that there is a minimum bound on the delay between a downward traveling ray received at the array and the subsequent ray that bounces off the seabed. This appears at roughly $0.36 \mathrm{~ms}$.

The CTD data was then processed using a linear fit, and is shown as the dot-dash line in Fig. 10. This line tracks better with the measured delays and shows culling at comparable ranges. Culling happens when an eigenray path disappears due to refraction. For example, the range at which the horizontally launched ray passes through the array depth corresponds to the maximum range at which the $\mathrm{D}$ eigenray (and thus its correlation with the B eigenray) exists.

As seen in Fig. 3, CTD data was recorded over the majority of the water column, but a few meters near the surface and seabed were not measured accurately. These sections were then populated by manually extrapolating to the boundaries using straight lines. The slope of these lines had a significant impact on the multipath structure. In Fig. 10, the "shallow surf" SSP uses a $-0.03 \mathrm{~m} / \mathrm{s}$ per $\mathrm{m}$ in the top $5 \mathrm{~m}$, whereas the "slope-surf" uses a more pronounced $-0.7 \mathrm{~m} / \mathrm{s}$ per $\mathrm{m}$ slope in the top $2 \mathrm{~m}$. A lower depth was used for the shallow surf SSP to prevent surface ducting, as this was not a phenomenon under investigation. Using the measured SSP causes better agreement with the acoustic data. The main effect of flattening the profile near the surface is increasing the distance that rays travel near the surface before being pulled down by the steeper gradient, causing ray culling to occur at greater ranges. The value of $-0.7 \mathrm{~m} / \mathrm{s}$ per $\mathrm{m}$ was found through manual adjustment to match the culling behavior observed in the acoustic data. It was also observed that lowering the source depth by up to $1 \mathrm{~m}$ had a minimal impact. It is evident that the cross beam output is highly sensitive to environment, and the water SSP in particular.

\section{Range estimation}

The previous section demonstrated that the multipath delay is a stable measurement, and this section shows how that can be used for target ranging. The measured SSP indicates that the waveguide is downward refracting and that the first pair of arrivals are culled at specific ranges. This brings up an ambiguity as it is not known solely from the measured time delay which pair of eigenrays are being correlated. However, modeling can help with this. Assuming the correct pair of eigenrays is chosen, the range follows by matching the measured time delay to modeled time delays computed over range. The assumption being made here is that time 

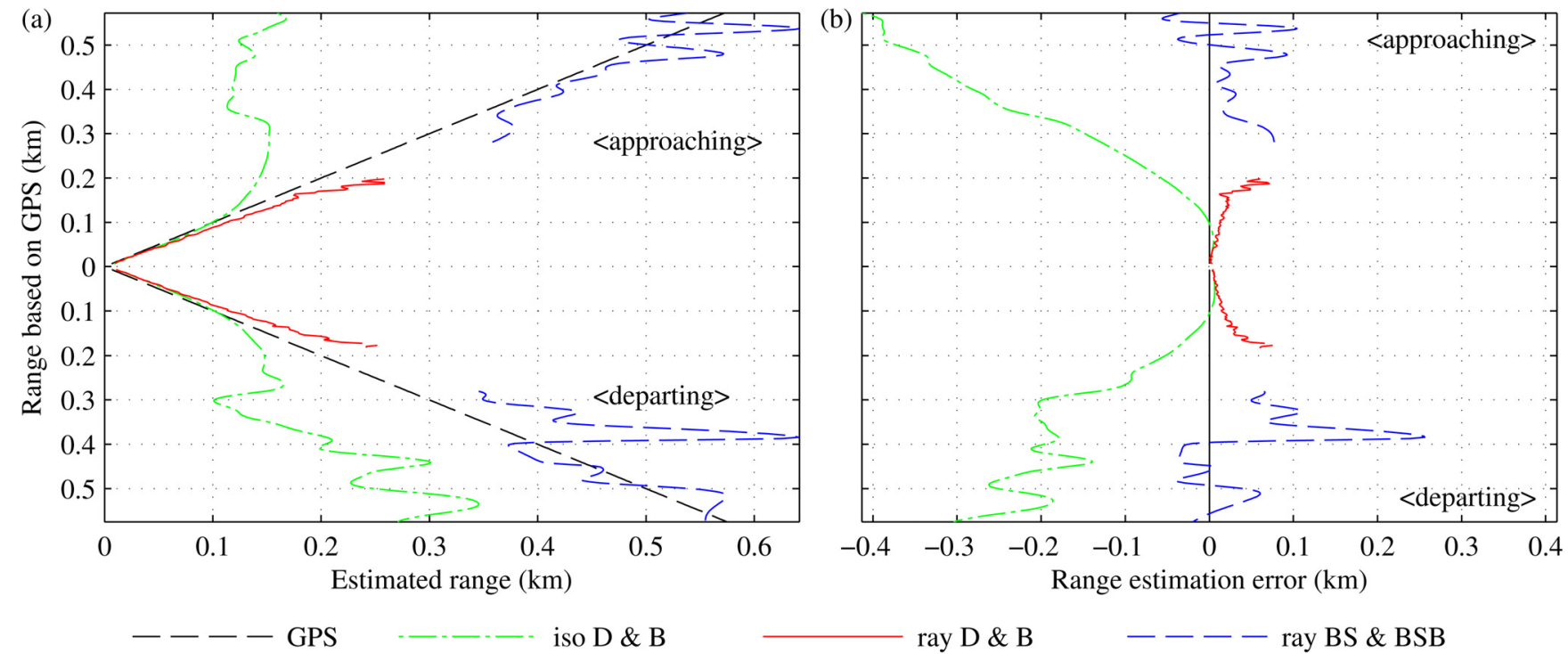

iso D \& B

ray $\mathrm{D} \& \mathrm{~B}$

-- ray BS \& BSB

FIG. 11. (Color online) (a) Range estimates for different water SSPs. (b) Errors in range estimates relative to GPS records. The "iso" line assumes a constant SSP (isovelocity) while the "ray" lines use the measured SSP and a ray tracer to predict delays from different pairs of eigenrays. Refraction effects cause the isovelocity model to breakdown at closer ranges while a ray model provides a significantly improved detection range.

delays for a single eigenray pair are monotonically decreasing as a function of range; and this is clearly observed in both modeled and measured curves in Fig. 9. However, this assumption may not be valid in regions with significant bathymetry variations.

The range inversion results are shown in Fig. 11(a). Errors relative to the GPS records are shown in Fig. 11(b). Comparing isovelocity and ray models, it can be seen that the effects of refraction cause the isovelocity model to start to break down between 100 and $200 \mathrm{~m}$, whereas the ray model provides reasonable estimates to over $500 \mathrm{~m}$ (over 22 water depths). Gaussian smoothing has been applied to the isovelocity and ray BS \& BSB curves as a visual aid to mitigate the measured and modeled variance of $\tau_{2,1}$. Nevertheless, the variance of the range estimates is observed to increase at more distant ranges. This is because the time delay measurement becomes less sensitive to changes in range when the target is farther away. Visually, this can be seen in Fig. 9 by the flattening out of both the measured and predicted time delays at greater ranges.

\section{CONCLUSIONS}

The technique presented in this article is a generalization of the passive fathometer to non-vertically traveling waves using an array geometry other than a vertical line. While this has been formulated in the context of target localization, the fundamental approach of using cross-beam correlation to process noise from a distant source is important because it provides a new way to measure acoustic propagation through a waveguide. Target localization is essentially a matter of interpreting measured multipath time delays and phases while properly accounting for propagation effects. However, the propagation effects may themselves be the object of study in future work. For example, it was observed that measurements were sensitive to the water sound speed profile, implying this can be used for ocean acoustic tomography. Also, the phase of the seabed reflection coefficient is a separate piece of information contained in the output, which may serve as a useful basis for performing geoacoustic inversion. Note that with this technique, the sound source is noise, such as from a passing boat.

Ray theory provides, perhaps, the most useful framework with which to understand the output of cross-beam correlation. It estimates a set of eigenrays that accurately describe and predict the measured quantities. In the experimental results, the strongest two eigenrays were measured out to about 35 water depths, but valid range estimates extended only to about 22 water depths. Environmental information proved to be important for accurately estimating more distant target ranges in which refraction effects were significant. The technique was demonstrated in a multitarget scenario (in which the acoustic data from two tracks were added) illustrating the ability of the compact volumetric array to handle off-bearing interference.

The kind of array used in this study is highly versatile and well suited for mobile platforms. Hence, this processing technique opens new possibilities for experimental designs. For example, AUVs that transect the water column could leverage depth-dependent environmental measurements to enhance on-board passive target localization capabilities.

\section{ACKNOWLEDGMENTS}

The authors would like to acknowledge support from the Centre for Maritime Research and Experimentation (CMRE), the Office of Naval Research (ONR), and Portland State University (PSU). We thank CMRE for designing and building the array, and for providing use of the NRV Alliance. We thank Steven Crocker and Jennifer Giard for assistance during the deployment. We also thank Charles Holland and Daniel Rouseff for helpful discussions.

Aulanier, F., Nicolas, B., Mars, J. I., Roux, P., and Brossier, R. (2013). "Shallow-water acoustic tomography from angle measurements instead of travel-time measurements,” J. Acoust. Soc. Am. 134, EL373-EL379. 
Badriasl, L., Dogancay, K., and Arulampalam, S. (2011). "3D passive localization in shallow water using bearing and multipath time-delay measurements," in Seventh International Conference on Intelligent Sensors, Sensor Networks and Information Processing (ISSNIP) (IEEE, New York), p. 473-478.

Capon, J. (1969). "High-resolution frequency-wave-number spectrum analysis," Proc. IEEE 57, 1408-1418.

Carter, G., and Knapp, C. (1976). "Time delay estimation," in IEEE International Conference on Acoustics, Speech, and Signal Processing (IEEE, New York), Vol. 1, pp. 357-360.

Gerstoft, P., Hodgkiss, W. S., Siderius, M., Huang, C.-F., and Harrison, C. H. (2008). "Passive fathometer processing," J. Acoust. Soc. Am. 123, $1297-1305$

Hahn, W. R. (1975). "Optimum signal processing for passive sonar range and bearing estimation," J. Acoust. Soc. Am. 58, 201-207.

Hamilton, M., and Schultheiss, P. M. (1992). "Passive ranging in multipath dominant environments. I. Known multipath parameters," IEEE Trans. Signal Processing 40,1-12.

Harrison, C. H. (2011). "The relation between the waveguide invariant, multipath impulse response, and ray cycles," J. Acoust. Soc. Am. 129, 2863-2877.

Harrison, C. H., and Siderius, M. (2008). "Bottom profiling by correlating beam-steered noise sequences," J. Acoust. Soc. Am. 123, 1282-1296.

Holland, C. W., and Osler, J. (2000). "High-resolution geoacoustic inversion in shallow water: A joint time- and frequency-domain technique," J. Acoust. Soc. Am. 107, 1263-1279.

Jensen, F. B., Kuperman, W. A., Porter, M. B., and Schmidt, H. (2011). "Ray methods," in Computational Ocean Acoustics, 2nd ed., edited by W. M. Hartmann (Springer New York, New York), Chap. 3, pp. 115-232.

Koch, R. A., and Knobles, D. P. (2005). "Geoacoustic inversion with ships as sources," J. Acoust. Soc. Am. 117, 626-637.

Lani, S. W., Sabra, K. G., Hodgkiss, W. S., Kuperman, W. A., and Roux, P. (2013). "Coherent processing of shipping noise for ocean monitoring," J. Acoust. Soc. Am. 133, EL108-EL113.

Leroy, C., Lani, S., Sabra, K. G., Hodgkiss, W. S., Kuperman, W. A., and Roux, P. (2012). "Enhancing the emergence rate of coherent wavefronts from ocean ambient noise correlations using spatio-temporal filters," J. Acoust. Soc. Am. 132, 883-893.

Mathias, D., Thode, A. M., Straley, J., and Andrews, R. D. (2013). "Acoustic tracking of sperm whales in the Gulf of Alaska using a two-element vertical array and tags," J. Acoust. Soc. Am. 134(3), 2446-2461.

Means, S. L., and Siderius, M. (2009). "Effects of sea-surface conditions on passive fathometry and bottom characterization," J. Acoust. Soc. Am. 126, 2234-2241.

Menon, R., and Gerstoft, P. (2013). "High resolution beamforming using 11 minimization," Proc. Meet. Acoust. 19, 1-4.
Nosal, E., and Neilfrazer, L. (2006). "Track of a sperm whale from delays between direct and surface-reflected clicks," Appl. Acoust. 67, 1187-1201.

Porter, M. B., and Bucker, H. P. (1987). "Gaussian beam tracing for computing ocean acoustic fields," J. Acoust. Soc. Am. 82, 1349-1359.

Rakotonarivo, S. T., and Kuperman, W. A. (2012). "Model-independent range localization of a moving source in shallow water," J. Acoust. Soc. Am. 132, 2218.

Rouseff, D., and Zurk, L. M. (2011). "Striation-based beamforming for estimating the waveguide invariant with passive sonar," J. Acoust. Soc. Am. 130, EL76-EL81.

Roux, P., Cornuelle, B. D., Kuperman, W. A., and Hodgkiss, W. S. (2008). "The structure of raylike arrivals in a shallow-water waveguide," J. Acoust. Soc. Am. 124, 3430-3439.

Sarkar, J., Cornuelle, B. D., and Kuperman, W. A. (2011). "Information and linearity of time-domain complex demodulated amplitude and phase data in shallow water," J. Acoust. Soc. Am. 130, 1242.

Siderius, M., Harrison, C. H., and Porter, M. B. (2006). "A passive fathometer technique for imaging seabed layering using ambient noise," J. Acoust. Soc. Am. 120, 1315-1323.

Siderius, M., Song, H., Gerstoft, P., Hodgkiss, W. S., Hursky, P., and Harrison, C. (2010). "Adaptive passive fathometer processing," J. Acoust. Soc. Am. 127, 2193-2200.

Song, H., Kuperman, W. A., Hodgkiss, W. S., Gerstoft, P., and Kim, J. S. (2003). "Null broadening with snapshot-deficient covariance matrices in passive sonar," IEEE J. Oceanic Eng. 28, 250-261.

Stotts, S. A., Koch, R. A., Joshi, S. M., Nguyen, V. T., Ferreri, V. W., and Knobles, D. P. (2010). "Geoacoustic inversions of horizontal and vertical line array acoustic data from a surface ship source of opportunity," IEEE J. Oceanic Eng. 35, 79-102.

Thode, A. M. (2000). "Source ranging with minimal environmental information using a virtual receiver and waveguide invariant theory," J. Acoust. Soc. Am. 108, 1582.

Tiemann, C. O., Thode, A. M., Straley, J., O'Connell, V., and Folkert, K. (2006). "Three-dimensional localization of sperm whales using a single hydrophone," J. Acoust. Soc. Am. 120, 2355-2365.

Van Trees, H. L. (2002a). "Correlated and coherent signals," in Optimum Array Processing, Detection, Estimation, and Modulation Theory, Part IV, 1st ed. (John Wiley and Sons, New York), Chap. 9.6, pp. 1233-1243.

Van Trees, H. L. (2002b). "Mismatched MVDR and MPDR beamformers," in Optimum Array Processing, Detection, Estimation, and Modulation Theory, Part IV, 1st ed. (John Wiley and Sons, New York), Chap. 6.6, pp. $488-513$.

Van Trees, H. L. (2002c). "Sample matrix inversion (SMI)," in Optimum Array Processing, Detection, Estimation, and Modulation Theory, Part IV, 1 st ed. (John Wiley and Sons, New York), Chap. 7.3, pp. 728-752.

Weston, D. E., and Stevens, K. J. (1972). "Interference of wide-band sound in shallow water," J. Sound Vib. 21, 57-64. 